\title{
In Individuals Following Aneurysmal Subarachnoid Haemorrhage, Hair Cortisol Concentrations Are Higher and More Strongly Associated with Psychological Functioning and Sleep Complaints than in Healthy Controls
}

\author{
Flora Colledge $^{a}$ Serge Brand ${ }^{a, b}$ Stefan Zimmerer ${ }^{c}$ Uwe Pühse ${ }^{a}$ \\ Edith Holsboer-Trachsler $^{\text {b }}$ Markus Gerber ${ }^{a}$ \\ ${ }^{a}$ Department of Sport, Exercise and Health, University of Basel, and ${ }^{\mathrm{b}}$ Center for Affective, Stress and Sleep Disorders, \\ Psychiatric Clinics of the University of Basel, Basel, Switzerland; ' University Hospital for Neurosurgery, University of \\ Graz, Graz, Austria
}

\section{Keywords}

Aneurysm · Depression · Neuroendocrinology · Health-related quality of life · Sleep

\begin{abstract}
Background: Following an aneurysmal subarachnoid haemorrhage (aSAH), many patients report persistent deficits in psychological functioning, characterised by high levels of stress and symptoms of depression, low life satisfaction, along with poor sleep. Such deficits have been associated with altered saliva and serum cortisol levels due to a dysregulation of hypothalamic-pituitary-adrenal axis activity (HPA-AA). However, hair cortisol concentrations (HCCs) have not been assessed in this population, although this method allows a long-term insight into cortisol values. Therefore, the objective of this study was to compare HCCs in aSAH patients and healthy controls and to examine how HCCs are associated with perceived stress, psychological functioning, and sleep complaints. Methods: In this cross-sectional study,
\end{abstract}

() 2017 S. Karger AG, Basel data on depressive symptoms, hypochondriacal beliefs, life satisfaction, and sleep complaints were gathered in $15 \mathrm{aSAH}$ patients and 17 healthy controls. HCCs of the previous 3 months were assessed. Results: aSAH patients had significantly higher HCCs than healthy controls. In aSAH patients, higher HCCs were significantly associated with increased depressive symptoms, hypochondriacal beliefs, lower life satisfaction, and increased sleep complaints. Such significant associations were not found in healthy controls. Conclusions: Our findings indicate that a dysregulation of HPA-AA is associated with some of the long-term impairments in psychological functioning and sleep in aSAH survivors. While the direction of association remained unclear, a dysregulated HPA-AA may be causally linked with the maintenance of poor psychological functioning and poor sleep. The overall findings should be considered in the planning of long-term treatment aimed at improving psychological functioning and sleep in aSAH patients.

(c) 2017 S. Karger AG, Basel
Prof. Dr. Markus Gerber

Division of Sport and Psychosocial Health, Sport Science Section Department of Sport, Exercise and Health, University of Basel Birsstrasse 320B, CH-4052 Basel (Switzerland)

E-Mail markus.gerber@ unibas.ch 


\section{Introduction}

Aneurysmal subarachnoid haemorrhage (aSAH) is characterised by the rupture of a cerebral artery and consequent bleeding into the area surrounding the brain known as the subarachnoid space [1]. Although this form of stroke has a high morbidity and mortality, in past decades improvements in neurosurgery and emergency treatment algorithms have increased the survival rate to $65 \%$ [2]. However, a large proportion of survivors continues to suffer from physical and psychological deficits following the initial recovery period [3]. The aSAH itself, combined with the frequently persistent deficits experienced in the aftermath, results in a severely impaired quality of life of aSAH survivors years after ictus [4].

As regards the psychological functioning, aSAH survivors reported increased anxiety levels [5], probably associated with the fear of recurrent haemorrhage [6]. Compared to healthy controls, aSAH patients also experienced more frequent depressive symptoms [7], increased fatigue [8], and a poorer sleep quality [7]. Symptoms of depression, fatigue, and poor sleep persisted up to 4 years after ictus [9], which explained why quality of life also remained low many months after surgery [10].

Besides the anxiety of a next stroke, one explanation for the persistently impaired psychological functioning in aSAH survivors might be the possibility of a dysregulation of hypothalamic-pituitary-adrenal axis activity (HPA-AA) after aSAH [11], with cortisol as main outcome marker. While a downregulation of the adrenocorticotropic hormone (ACTH), the precursor of cortisol, has been repeatedly shown, findings on the cortisol levels are less consistent.

With regard to ACTH, an underactivity of the HPAAA has been documented in aSAH survivors: Tanriverdi et al. [12] reported ACTH deficiencies in $23 \%$ of aSAH patients in the acute phase, and $14 \% 12$ months after ictus. Moreover, in a large register study with $417 \mathrm{aSAH}$ patients the prevalence of hypopituitarism in the chronic phase (at least 5 months after the event) by laboratory values, physician diagnoses, and stimulation tests, was 35 , 36 , and $70 \%$, respectively, with decreased ACTH levels being the most frequent endocrine dysfunction in this group of patients [13]. Finally, similar results were reported in a meta-analysis with 19 studies and 1,137 aSAH patients, yielding hypopituitarism in $47 \%$ of patients in the chronic phase [14].

As regards the cortisol secretion assessed in blood and saliva, prior research has provided mixed results. For instance, Karaca et al. [15] found that serum basal cortisol

Hair Cortisol in aSAH Patients levels were significantly elevated 3 years after aSAH. However, Brand et al. [7] found no statistically significant differences between aSAH patients (5-9 months after ictus) and healthy controls with regard to the salivary cortisol awakening response (CAR), neither for the area under the curve with respect to increase (AUCi; representing the increase in cortisol secretion in relation to the first of the postwaking measurements) nor with respect to ground (AUCg; representing overall cortisol output). Furthermore, the CAR was neither related to patients' psychological functioning nor to their subjective or objective sleep. By contrast, Gerber et al. [16] observed that compared to healthy controls, aSAH patients (mean $=44$ months after ictus) had significantly higher salivary CAR as measured by the AUCi. However, aSAH patients also had descriptively lower overall cortisol output values, represented by the AUCg. Gerber et al. concluded that a disconnection between the AUCi and AUCg values was plausible, as the values represented differing aspects of HPA-AA (reactivity vs. total output) [16].

In summary, previous research suggested that the HPA-AA was indeed affected in aSAH patients. However, given the inconsistencies in the available literature, it remains unclear whether aSAH patients have lower or higher basal cortisol levels than healthy controls, and how exactly cortisol levels are related to other health outcomes. In part, the inconsistencies found in prior research can be attributed to the fact that salivary and plasma cortisol levels represent single time point assessments and only reveal acute circulating cortisol levels or mean cortisol secreted over a relatively short period of time. Moreover, salivary and plasma cortisol are easily affected by temporary or transient disturbances in psychosocial stress on the day of measurement, subject to diurnal variations and affected by any food intake, smoking and physical activity that takes place shortly before sampling. By contrast, hair cortisol measurement has the potential to overcome these methodological difficulties associated with the assessment of long-term cortisol levels $[17,18]$. For instance, Stalder et al. [19] showed that hair cortisol concentrations (HCCs) have a high intra-individual stability, with correlations of repeated assessments between 2 months and 1 year ranging between 0.68 and 0.79 . Therefore, hair cortisol assessments have become an increasingly accepted approach in endocrinology and biopsychology during the last few years [18].

To date, hair cortisol as a biomarker of HPA-AA has not been studied in aSAH patients. Therefore, the goal of the present study was twofold: first, to compare for the first time HCCs in aSAH patients and healthy controls;

Neuropsychobiology 2017;75:12-20 DOI: $10.1159 / 000477966$ 
second, to examine how HCCs are associated with perceived stress, psychological functioning, and sleep complaints, domains in which aSAH patients have frequently reported deficits, separately for aSAH patients and healthy controls.

The following 2 hypotheses were formulated. Following Dettenborn et al. [20] and Van Uum et al. [21], our first hypothesis was that higher HCCs would be associated with poorer psychological functioning (including depressive symptoms, hypochondria, and life satisfaction). Second, although Maurer et al. [22] were unable to find significant associations between hair cortisol and objective sleep parameters in young children, we assumed that higher HCCs would be associated with poorer quality of sleep in aSAH survivors, because poor sleep and depressive symptoms are strongly correlated in aSAH patients $[16,23]$.

\section{Methods}

\section{Participants}

The sample comprised 32 participants (22 women, 10 men; mean age $=57.4$ years, $\mathrm{SD}=10.7$; mean $\mathrm{BMI}=26.2, \mathrm{SD}=3.7$ ). Fifteen participants ( 11 women, 4 men; mean age $=57.2$ years, $\mathrm{SD}=8.9$ ) were patients with aSAH and following neurosurgical or endovascular intervention. One patient had received coiling, and 14 underwent clipping. No patient presented with additional untreated aneurysms. The mean Glasgow Outcome Score was $4.33(\mathrm{SD}=0.6)$, indicating an ability to live independently but with some deficits. Five had a Fisher grade of 4 , another 5 had a grade of 3 , and 1 of 2 . These data were not available for 4 patients. Five had a Hunt and Hess grade of 4, then 5 had a grade of 3 , and 2 of 2. This information was not available for 3 patients. The mean time spent in intensive care was 8.3 days $(S D=2.8)$. The mean time after intervention was 44 months $(S D=28.9$; range $3-72$ months, median $=60$ months, 25 th percentile: 9 months, 75 th percentile: 69 months).

The second group consisted of 17 healthy controls ( 11 women, 6 men; mean age $=59.8$ years, $S D=10.8)$. To reduce bias, healthy controls were matched for age and sex. Accordingly, the 2 groups did not differ with regard to age, $F(1,31)=0.01, p=0.946$, $\eta^{2}=0.000$, sex, $\chi^{2}(1,31)=0.28, p=0.599, \Phi=0.092$, and BMI, $F(1,31)=2.75, p=0.108, \eta^{2}=0.084$. Use of medication tended to be more frequent among aSAH patients $(n=12 ; 80 \%)$ than healthy controls $(n=3 ; 25 \%), \chi^{2}(1,31)=3.69, p=0.055, \Phi=0.322$. However, none of the participants indicated that they used corticosteroid medication.

\section{Study Design and Procedure}

The design of this study was cross-sectional. Patients were eligible if they were treated for aSAH at the Department of Neurosurgery at the University Hospital Basel, Switzerland, and were recruited during the consultation hours. Healthy controls were recruited via online advertisements on local news websites. Those willing to participate in the study provided informed consent and were assured confidentiality of their responses prior to the data assessment. Approval for the study was obtained from the local ethical committee (EKNZ, approval No. 61/12). The study was conducted in accordance with the ethical principles described in the 1964 Declaration of Helsinki. Power analysis was used to establish the minimal sample size to find large correlations $(r=0.50-0.60)$. Using G*Power Software 3 (alpha error $=0.05$, power $=0.80$ ), between $13(r=0.60)$ and $21(r=0.50)$ aSAH patients and healthy controls were needed.

All data assessments took place at the participants' homes between April 2012 and April 2013 and were carried out by a trained research assistant. Previously, data from this study had been published indicating that aSAH patients reported poorer subjective sleep and more sleep-related dysfunctional cognitions and hypochondriacal beliefs than healthy controls [16]. However, data on hair cortisol have not been published elsewhere.

\section{Instruments}

Depressive Symptoms

The Beck Depression Inventory was used to assess the severity of depressive symptoms [24]. It consists of 21 items scaled from 0 to 3 including a range of affective, behavioural, cognitive, and somatic symptoms that are indicative of unipolar depression (e.g., "I am so unhappy/sad that I can't stand it"). Possible scores ranged from 0 to 63 with higher scores indicating more depressive symptoms.

Hypochondriacal Beliefs

The 14-item Whiteley Index was used to assess hypochondriacal beliefs (e.g., "Are you bothered by many aches and pains?") [25]. Answers were "yes" $(=1)$ or "no" $(=0)$, with higher sum scores reflecting a greater inclination to overestimate bodily sensations and pain.

\section{Satisfaction with Life}

The 5-item Satisfaction with Life Scale was used to obtain an overall judgement of respondents' satisfaction with life [26]. Answers are given on a 7-point Likert-type scale (e.g." "In most ways, my life is close to my ideal"). The items were summed to generate a composite score.

\section{Sleep Complaints}

The 7-item Insomnia Severity Index was administered to assess participants' subjective sleep complaints [27]. The items refer in part to the DSM-IV criteria for insomnia by measuring difficulty in falling asleep, difficulties maintaining sleep, early morning awakening, increased daytime sleepiness, low daytime performance, low satisfaction with sleep, and worrying about sleep. Possible answers range from 0 (not at all) to 4 (very much), with higher sum scores reflecting higher insomnia.

\section{Hair Cortisol}

HCC was assessed using samples of hair, approximately $3 \mathrm{~mm}$ in diameter, which are cut close to the scalp of the study participants. The $3 \mathrm{~cm}$ of hair closest to the scalp are tested for cortisol concentration and provide a value encompassing the previous 3 months. In some cases, participants with particularly short hair do not provide sufficient material for analysis; this was the case with 3 participants from the control group, who were subsequently excluded from the analyses. The samples were analysed at the bio- 
Table 1. Descriptive statistics of all study variables

\begin{tabular}{|c|c|c|c|c|c|c|}
\hline & \multirow[t]{2}{*}{ Mean \pm SD } & \multirow[t]{2}{*}{$\alpha$ (items) } & \multicolumn{2}{|l|}{ Range } & \multirow[t]{2}{*}{ Skew } & \multirow[t]{2}{*}{ Kurtosis } \\
\hline & & & potential & actual & & \\
\hline Hair cortisol concentration, $\mathrm{nmol} / \mathrm{L}$ & $3.28 \pm 1.80$ & - & $0+$ & $0.74-10.38$ & 1.80 & 2.99 \\
\hline Depressive symptoms (BDI) & $6.47 \pm 5.48$ & $0.87(21)$ & $0-63$ & $0-26$ & 1.56 & 3.83 \\
\hline Hypochondriacal beliefs (WI) & $3.13 \pm 3.93$ & $0.78(14)$ & $0-56$ & $0-19$ & 2.68 & 8.35 \\
\hline Satisfaction with life (SWLS) & $26.59 \pm 7.00$ & $0.93(5)$ & $5-35$ & $5-35$ & -1.41 & 1.95 \\
\hline Sleep complaints (ISI) & $5.91 \pm 4.50$ & $0.83(7)$ & $0-28$ & $0-17$ & 0.90 & 0.07 \\
\hline
\end{tabular}

$\alpha=$ Cronbach's alpha. $n=32$. BDI, Beck Depression Inventory; WI, Whiteley Index; SWLS, Satisfaction with Life Scale; ISI, Insomnia Severity Index.

chemical laboratory of the University of Dresden, Germany, as described in the protocol by Kirschbaum et al. [28]. To summarise, each individual hair sample is washed twice with $2.5 \mathrm{~mL}$ of isopropanol for 3 min, dried over a 12 -h period, and then powdered in a Retsch ball mill at $30.0 \mathrm{~Hz}$, for a period of $5 \mathrm{~min} ; 25 \mathrm{mg}$ of the powdered hair is then transferred into a $2-\mathrm{mL}$ cryovial. Then 1.5 $\mathrm{mL}$ of pure methanol is added to the vial, which is rotated slowly for $45 \mathrm{~min}$, in order for steroids to be extracted. The sample is then centrifuged at 10,000 r.p.m. for a period of $3 \mathrm{~min}$ (relative centrifugal force $=10,080 \mathrm{~g}$ ). One millilitre of clear supernatant is placed in a new vial, and the methanol is evaporated using a steady stream of nitrogen $\left(60^{\circ} \mathrm{C}\right)$ until the sample is dry; $0.4 \mathrm{~mL}$ of commercially available phosphate buffer (IBL-International, Hamburg, Germany) containing $0.1 \%$ bovine serum albumin, at a $\mathrm{pH}$ of 7.1 , is added to the vial, which is then vortexed for $15 \mathrm{~s}$. Cortisol is determined by analysing $80 \mu \mathrm{L}$ of the reconstituted sample by a commercially available immunoassay (CLIA, IBL-International, Hamburg, Germany). In accordance with the study of Skoluda et al. [29], the minimal amount of powdered hair used in this study was $25 \mathrm{mg}$, instead of $50 \mathrm{mg}$ in the protocol of Kirschbaum et al. [28]. Intra- and interassay coefficients of variance were below $10 \%$ in the present study.

\section{Statistical Analyses}

None of the participants had missing values. Differences in HCCs, psychological functioning, and sleep between aSAH patients and healthy controls were examined with univariate analyses of variances (ANOVAs). Differences between aSAH patients and healthy controls with regard to stress, psychological functioning, and sleep have been described elsewhere [16]. Bivariate associations between hair cortisol levels, stress, psychological functioning, and sleep were examined via Pearson's product moment correlations, separately for aSAH patients and healthy controls. Following Cohen [30], effect sizes for ANOVAs (partial $\eta^{2}$ ) were considered as follows: small $=0.01>\eta^{2}<0.059$, medium $=$ $0.06>\eta^{2}<0.139$, or large $=\eta^{2} \geq 0.14$. Cohen's criteria were also used to interpret correlations (e.g., small: 0.10-0.30; medium: $0.30-0.50$; large: $>0.50$ ) [30]. The level of probability was set at $p<0.05$ across all analyses. All statistics were performed with SPSS $^{\circledR} 22.0$ (IBM Corporation, Armonk, NY, USA) for Apple MacIntosh ${ }^{\circledR}$.

Hair Cortisol in aSAH Patients

\section{Results}

\section{Descriptive Statistics}

The descriptive statistics for the total sample are presented in Table 1. Table 1 also shows that the internal consistencies for all indicators related to psychological functioning and subjective sleep were adequate.

\section{Differences in Hair Cortisol, Psychological}

Functioning, and Sleep between aSAH Patients and

\section{Healthy Controls}

HCCs were significantly higher in aSAH patients compared to healthy controls, $F(1,28)=5.04, p<0.05, \eta^{2}=$ 0.157 (aSAH: mean $=4.24 \mathrm{nmol} / \mathrm{L}, \mathrm{SD}=2.91$; healthy controls: mean $=2.39 \mathrm{nmol} / \mathrm{L}, \mathrm{SD}=1.26)$. Moreover, aSAH patients perceived more depressive symptoms than healthy controls, $F(1,28)=4.75, p<0.05, \eta^{2}=0.149$ (aSAH: mean $=8.50, \mathrm{SD}=6.65$; healthy controls: mean $=$ $4.27, \mathrm{SD}=3.41$ ), reported higher hypochondriacal beliefs, $F(1,28)=6.72, p<0.05, \eta^{2}=0.199(\mathrm{aSAH}:$ mean $=4.00$, $\mathrm{SD}=3.44$; healthy controls: mean $=1.60, \mathrm{SD}=0.99)$, and exhibited more sleep complaints, $F(1,28)=10.04, p<$ $0.01, \eta^{2}=0.271$ (aSAH: mean $=8.64, \mathrm{SD}=5.15$; healthy controls: mean $=3.93, \mathrm{SD}=2.49$ ). No significant group differences were found with regard to satisfaction with life, $F(1,28)=1.60, p=0.22, \eta^{2}=0.056$ (aSAH: mean $=$ $24.93, \mathrm{SD}=8.16$; healthy controls: mean $=28.27, \mathrm{SD}=$ 5.96).

\section{Correlations between Hair Cortisol and Psychological Functioning}

In aSAH patients, higher HCCs were significantly associated with increased depressive symptoms, hypochondriacal beliefs, and with lower life satisfaction (Table 2). The scatterplot shown in Figure 1 suggests that the statis-

Neuropsychobiology 2017;75:12-20 DOI: $10.1159 / 000477966$ 
Table 2. Correlations between hair cortisol and psychological variables, separately for aSAH patients and healthy controls

\begin{tabular}{llllrrr}
\hline & & 1 & 2 & 3 & \multicolumn{2}{c}{4} \\
\hline 1 & Hair cortisol concentration, nmol/L & - & -0.40 & -0.13 & -0.10 & -0.26 \\
2 & Hypochondriacal beliefs (WI) & $0.62^{*}$ & - & 0.20 & 0.07 & 0.40 \\
3 & Depressive symptoms (BDI) & $0.56^{*}$ & $0.74^{* * *}$ & - & -0.39 & 0.27 \\
4 & Satisfaction with life (SWLS) & $-0.65^{*}$ & $-0.65^{* *}$ & $-0.72^{* *}$ & - & -0.29 \\
5 & Sleep complaints (ISI) & $0.54^{*}$ & 0.18 & $0.56^{*}$ & -0.26 & - \\
\hline
\end{tabular}

Above the diagonal: healthy controls $(n=17)$. Below the diagonal: aSAH patients $(n=15) .{ }^{* * *} p<0.001$, ** $p<0.01, * p<0.05$. For abbreviations, see Table 1 .

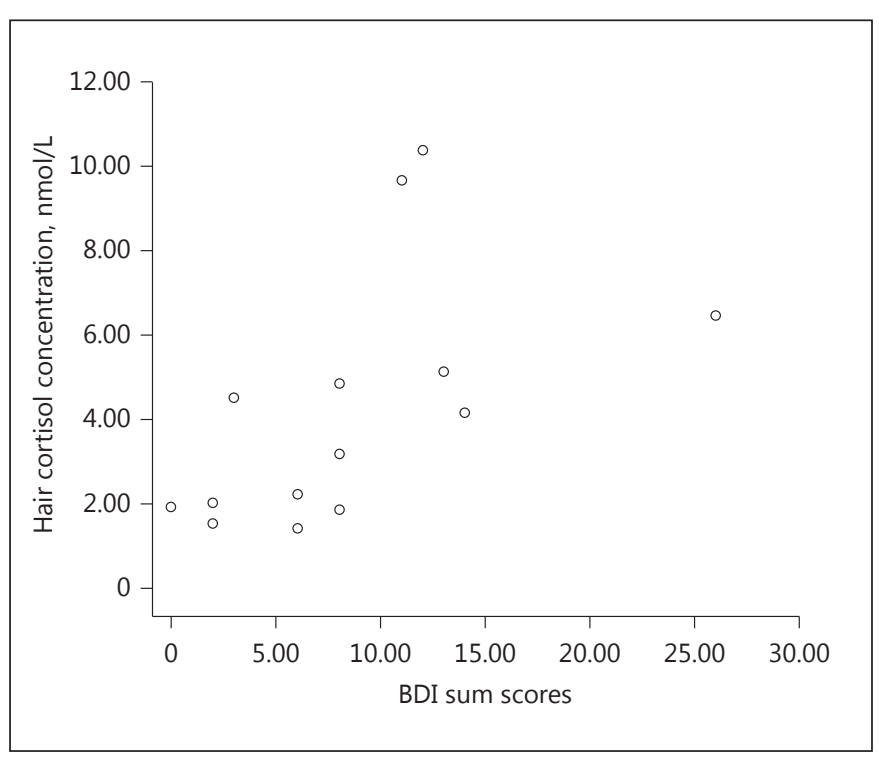

Fig. 1. Scatterplot illustrating the bivariate relationship between hair cortisol concentrations and depressive symptoms in aSAH patients.

tically significant correlation between patients' HCCs and their depressive symptoms was not due to the impact of a single outlier. Similar patterns of results were found for all other indicators of psychological functioning.

In healthy controls, none of the correlations were significant. Descriptively, however, higher HCCs tended to be associated with fewer psychological symptoms ( $r=$ -0.08 to $-0.40, p=\mathrm{ns}$ ).

\section{Correlations between Hair Cortisol and Sleep}

In aSAH patients, higher HCCs were significantly associated with increased sleep complaints (Table 2). In contrast, in healthy controls, no significant associations existed between participants' HCCs and their subjective sleep. However, descriptively, higher HCCs were associated with fewer subjective sleep complaints $(r=-0.26$, $p=\mathrm{ns})$.

\section{Discussion}

The key findings of the present study are that aSAH patients had higher hair cortisol levels than healthy controls. Moreover, in the aSAH group, statistically significant associations existed between increased HCCs and impaired psychological functioning and sleep, while such associations were not observed in healthy controls. In many respects, this study explored unknown territory. To the best of our knowledge, no research has been published so far on hair cortisol levels in aSAH patients compared to healthy controls, and few studies have assessed the degree to which high hair cortisol levels are associated with stress and psychological functioning in clinical and nonclinical populations. Thus, the present study expands upon the current literature in an important way, in that we were able to show that patients with aSAH reported poorer subjectively assessed psychological functioning and more sleep complaints [16], which at the physiological level were reflected by statistically significantly higher HCCs. Higher HCCs, in turn, are believed to reflect a chronic upregulation of HPA-AA.

An underlying cause of irregularities in endocrine dysfunction in patients who have undergone aSAH and subsequent medical intervention can be damage to regions of the brain involved in HPA axis regulation $[31,32]$. These irregularities have been shown to persist up to 24 months after ictus [33]. Some studies have suggested that this is
16

Neuropsychobiology 2017;75:12-20 DOI: $10.1159 / 000477966$
Colledge/Brand/Zimmerer/Pühse/ Holsboer-Trachsler/Gerber 
more likely following clipping, as opposed to coiling [34]; in the present study, 14 of the 15 patients had undergone clipping. For instance, damage to the hippocampus, which can be the result of clipping, can lead to alterations in cortisol secretion [35]. However, in the present study, there was no suggestion that major areas of the hypothalamic-pituitary system were damaged. Consequently, we are unable to draw conclusions about the influence of particular haemorrhage locations on HCCs.

Given the absence of previous studies, we did not formulate a specific hypothesis as to whether aSAH patients would have higher or lower HCCs compared to healthy controls. In the present study, we observed higher HCCs in the aSAH group. Accordingly, the present data are at odds with studies showing that patients with aSAH have suffered from hypopituitarism $[14,36]$ and tended to have a lower morning overall cortisol output [16]. However, previous studies have also shown that compared to healthy controls, aSAH patients had a higher CAR as measured by the AUCi [16]. Moreover, serum basal cortisol and ACTH levels were found to significantly increase from the first to the third years after surgical intervention [15]. Thus, the elevated HCCs might be explained by the fact that the mean time since surgery amounted to 44 months in the present study. Unfortunately, the present data do not allow a determination of the cause of the high HCCs found in aSAH patients. Thus, once these findings have been confirmed in larger samples, a next step could be to examine whether the elevated HCCs in aSAH survivors are attributable to ACTH dependent or ACTH independent sources [37]. Nevertheless, the elevated HCCs in aSAH patients are critical as increased HCCs have been shown to constitute a risk factor for cardiovascular diseases [38], diabetes mellitus [39], increased BMI and obesity [40], and the occurrence of metabolic syndrome [41]. Finally, as reported in a previous paper [16], aSAH patients reported significantly higher depressive symptoms, hypochondriacal beliefs, and sleep complaints than healthy controls.

Two hypotheses were formulated. Our first hypothesis was that higher HCCs would be associated with poorer psychological functioning, and the data did confirm this notion, though specifically in aSAH patients, but not in healthy controls. Importantly, in aSAH patients, most correlations between HCCs and psychological functioning were of strong magnitude ( $r_{s}$ between 0.54 and -0.65 ), and the inspection of the scatterplots indicated that single outliers did not affect the correlations. Moreover, the same pattern of results was found across all indicators of psychological functioning including depressive symp-

Hair Cortisol in aSAH Patients toms, hypochondriacal beliefs, and life satisfaction. The close link between increased HCCs and poor psychological functioning accords well with previous research showing that higher HCCs have been observed in individuals suffering from depression [20] and severe chronic pain [21]. The fact that HCCs were significantly associated with psychological functioning in aSAH patients, and not in healthy controls, accords well with a study on healthy young adults [42], in which higher HCCs were associated with lower depressive symptoms. However, our findings add to the existing literature by providing preliminary evidence that high HCC levels can be used as a biomarker for poor psychological functioning among aSAH populations. This finding is important as researchers were not able to correlate assessments of pituitary hormone dysregulation with the outcomes of aSAH patients in previous studies [36].

Our second hypothesis was that higher HCCs would be associated with more frequent sleep complaints [16, 23]. The data supported this hypothesis for aSAH patients, but not for healthy controls. While heightened activity of the HPA axis has been associated with sleep complaints [43], it has to date not been conclusively established whether HPA axis overactivity precedes sleep complaints or occurs as a result of the emotional stress of poor sleep [44].

While the present study provided novel insights into the association between HCCs, psychological functioning, and sleep in aSAH versus healthy controls, the current results must be considered in light of certain limitations. First, the relatively small sample size made it difficult to detect statistically significant correlations, and precluded separate analyses for male and female participants. Nevertheless, results showed medium to large effect sizes, which do not rely on sample sizes. Second, patients were recruited at different time points following surgery. However, ANOVAs comparing participants below versus above the median of posttreatment time revealed no influence on the existence or severity of deficits in the measured parameters or with regard to HCC (data not shown). Accordingly, the increased hair cortisol levels in aSAH patients appear to be due to the event per se and do not represent a cumulative effect over time. Third, the cross-sectional study design did not allow conclusions to be drawn regarding a possible causal relationship between HCCs, psychological functioning, and sleep. Fourth, medication intake prior to the beginning of the study was not recorded. It is therefore possible that the hair samples may have been affected by medications which influence cortisol secretion. However, as noted in

Neuropsychobiology 2017;75:12-20 DOI: $10.1159 / 000477966$ 
the text, participants provided detailed information about their medication intake at the moment of the data assessment. The inspection of this data revealed that no participant consumed corticosteroid medication. Moreover, the medications consumed have been analysed in light of their potential effects on the activity of the HPA axis and cortisol secretion. In the aneurysm group, 1 patient took the diabetes medication metformin. While Haugen [45] notes that metformin has been reported to affect thyroid function, this finding has not been confirmed by multiple studies and requires further investigation. Moreover, no direct effects upon cortisol secretion have been documented [46]. Additionally, 1 patient took 2 forms of antidepressant (escitalopram and mirtazapine), which both have been shown to reduce salivary cortisol $[47,48]$. While purely hypothetical, as our findings show increased cortisol levels amongst aSAH patients, we feel that the intake of these medications by 1 patient is not likely to have exaggerated our results. In the control group, 1 participant took medication for high cholesterol (Crestor), and 1 took hormone replacement therapy. In the comprehensive list of medications of Granger et al. [49], which, by their mechanism of action, could influence salivary cortisol, these forms of medication are considered. However, Rosuvastatin, the active ingredient in Crestor, taken alone, has not been shown to affect plasma cortisol levels $[50,51]$. Regarding the hormone replacement therapy, this was a combination of oestrogen and progesterone, which has not been shown to significantly increase plasma cortisol concentration, in comparison to oestrogen alone [52]. Fifth, we acknowledge that while we used a standardised and validated tool to assess self-reported symptoms of depression, this instrument does not correspond to a diagnostic tool to assess depression as defined in the international classification systems such as the ICD-10 [53]. Because the HPA system is usually disturbed during an acute major depressive episode [54], we were not able to control for this confounding factor of altered HPA regulation.

\section{Conclusion}

The findings indicate that a dysregulation of HPA-AA may explain some of the long-term deficits experienced by aSAH survivors. However, these findings need to be replicated in future research, and more knowledge is required as to how hair cortisol levels change over the course of time in aSAH survivors. Finally, our findings suggest that HPA axis abnormalities should be taken into account in the planning of long-term treatment in aSAH survivors.

\section{Acknowledgement}

This study was funded by the Freiwillige Akademische Gesellschaft, Basel, Switzerland. The funding source had no involvement in study design, data collection, analysis, and interpretation, article preparation, or the decision to submit the article for publication.

\section{Disclosure Statement}

The authors declare that there is no conflict of interest.

\section{References}

1 Suarez JI, Tarr RW, Selman WR: Aneurysmal subarachnoid hemorrhage. N Engl J Med 2006;354:387-396.

2 Rinkel GJ, Algra A. Long-term outcomes of patients with aneurysmal subarachnoid haemorrhage. Lancet 2011;10:349-356.

3 Connolly ES, Rabinstein AA, Carhuapoma JR, Derdeyn CP, Dion J, Higashida RT, Hoh BL, Kirkness CJ, Naidech AM, Ogilvy CS, Patel AB, Thompson BG, Vespa P: Guidelines for the management of aneurysmal subarachnoid hemorrhage: a guideline for healthcare professionals from the American Heart Association/American Stroke Association. Stroke 2012;43:1711-1737.
4 Passier PE, Visser-Meily JMA, Rinkel GJE, Lindeman E, Post MWM: Life satisfaction and return to work after aneurysmal subarachnoid hemorrhage. J Stroke Cerebrovasc Dis 2011;20:324-329.

5 Powell J, Kitchen N, Heslin J, Greenwood R: Psychosocial outcomes at 18 months after good neurological recovery from aneurysmal subarachnoid haemorrhage. J Neurol Neurosurg Psychiatry 2004;75:1119-1124.

6 Hütter BO, Kreitschmann-Andermahr I: Subarachnoid hemorrhage as a psychological trauma. J Neurosurg 2014;120:923-930.
7 Brand S, Zimmerer S, Kalak N, von Planta S, Schwenzer-Zimmerer K, Müller A, Zeilhofer $\mathrm{H}$, Holsboer-Trachsler E: Compared to controls, patients with ruptured aneurysm and surgical intervention show increase in symptoms of depression and lower cognitive performance, but their objective sleep is not affected. World J Biol Psychiatry 2015;16:96-105.

8 Kutlubaev MA, Barugh AJ, Mead GE: Fatigue after subarachnoid haemorrhage: a systematic review. J Psychosom Res 2012;72:305310.

9 Visser-Meily JM, Rhebergen ML, Rinkel GJ, van Zandvoort MJ, Post MW: Long-term health-related quality of life after aneurysmal subarachnoid hemorrhage: relationship with psychological symptoms and personality characteristics. Stroke 2009;40:1526-1529.

Colledge/Brand/Zimmerer/Pühse/ Holsboer-Trachsler/Gerber 
10 Passier PE, Visser-Meily JM, Rinkel GJ, Lindeman E, Post MW: Determinants of healthrelated quality of life after aneurysmal subarachnoid hemorrhage: a systematic review. Qual Life Res 2013;22:1027-1043.

11 Shin IY, Joo HM, Chung YG, Kim MS, Park JW, Ahn RS: Abnormal diurnal pattern of cortisol secretion in patients after aneurysmal subarachnoid hemorrhage. Stress 2011;14: 156-165.

12 Tanriverdi F, Dagli AT, Karaca Z, Unluhizarci K, Selcuklu A, Casanueva FF, Kelestimur F: High risk of pituitary dysfunction due to aneurysmal subarachnoid haemorrhage: a prospective investigation of anterior pituitary function in the acute phase and 12 months after the event. Clin Endocrinol 2007;67:931937.

13 Schneider HJ, Schneider M, KreitschmannAndermahr I, Tuschy U, Wallaschofski H, Fleck S, Faust M, Renner CI, Kopczak A, Saller B, Buchfelder M, Jordan M, Stalla GK: Structured assessment of hypopituitarism after traumatic brain injury and aneurysmal subarachnoid hemorrhage in 1,242 patients: the German interdisciplinary database. J Neurotraum 2011;28:1693-1698.

14 Schneider HJ, Kreitschmann-Andermahr I, Ghigo E, Stalla GK, Agha A: Hypothalamopituitary dysfunction following traumatic brain injury and aneurysmal subarachnoid hemorrhage: a systematic review. JAMA 2007;298: 1429-1438.

15 Karaca Z, Tanriverdi F, Dagli AT, Selcuklu A, Casanueva FF, Unluhizarci K, Kelestimur F: Three years prospective investigation of pituitary functions following subarachnoid haemorrhage. Pituitary 2013;16:76-82.

16 Gerber M, Colledge F, Pühse U, HolsboerTrachsler E, Zimmerer S, Brand S: Sleep quality, sleep EEG pattern, mental well-being and cortisol secretion in patients with ruptured aneurysm post-treatment: a comparison with post-surgery meningioma patients and controls. Neuropsychobiology 2016;73:148-159.

17 Russell E, Koren G, Rieder M, Van Uum S: Hair cortisol as a biological marker of chronic stress: current status, future directions and unanswered questions. Psychoneuroendocrinology 2012;37:589-601.

18 Stalder T, Kirschbaum C: Analysis of cortisol in hair - state of the art and future directions. Brain Behav Immun 2012;26:1019-1029.

19 Stalder T, Steudte S, Miller R, Skoluda N, Dettenborn L, Kirschbaum C: Intraindividual stability of hair cortisol concentrations. Psychoneuroendocrinology 2012;37:602-610.

20 Dettenborn L, Muhtz C, Skoluda N, Stalder T, Steudte S, Hinkelmann K, Kirschbaum C, Otte C: Introducing a novel method to assess cumulative steroid concentrations: increased hair cortisol concentrations over 6 months in medicated patients with depression. Stress 2012;15:348-353.
21 Van Uum S, Sauvé B, Fraser LA, Morley-Forster P, Paul TL, Koren G: Elevated content of cortisol in hair of patients with severe chronic pain: a novel biomarker for stress. Stress 2008; 11:483-488.

22 Maurer N, Perkinson-Gloor N, Stalder T, Hagmann-von Arx P, Brand S, HolsboerTrachsler E, Wellmann S, Grob A, Weber P, Lemola S: Salivary and hair glucocorticoids and sleep in very preterm children during school age. Psychoneuroendocrinology 2016; 72:166-174.

23 Brand S, Gerber M, Pühse U, HolsboerTrachsler E: Depression, hypomania and dysfunctional cognitions as mediators between stress and insomnia: the best advice is not always found on the pillow! Int J Stress Management 2010;17:114-134.

24 Beck AT, Ward CH, Mendelson M, Mock J, Erbaugh J: An inventory for measuring depression. Arch Gen Psychiatry 1961;4:561571.

25 Pilowsky I: Dimensions of hypochondriasis. Br J Psychiatry 1967;113:89-93.

26 Diener E, Emmons RA, Larsen RJ, Griffin S: The satisfaction with life scale. J Pers Assess 1985;49:71-75.

27 Bastien CH, Vallières A, Morin CM: Validation of the Insomnia Severity Index (ISI) as an outcome measure for insomnia research. Sleep Med 2001;2:297-307.

28 Kirschbaum C, Tietze A, Skoluda N, Dettenborn L: Hair as a retrospective calendar of cortisol production - increased cortisol incorporation into hair in the third trimester of pregnancy. Psychoneuroendocrinology 2009; 34:32-37.

29 Skoluda N, Dettenborn L, Stalder T, Kirschbaum C: Elevated hair cortisol concentrations in endurance athletes. Psychoneuroendocrinology 2012;37:611-617.

30 Cohen J: Statistical Power Analysis for the Behavioral Sciences. Mahwah, Erlbaum, 1988.

31 Schneider HJ, Kreitschmann-Andermahr I, Ghigo E, Stalla GK, Agha A: Hypothalamopituitary dysfunction following traumatic brain injury and aneurysmal subarachnoid hemorrhage: a systematic review. JAMA 2007;298: 1429-1438.

32 Aimaretti G, Ambrosio MR, Di Somma C, Fusco A, Cannavo S, Gasperi M, Scaroni C, De Marinis L, Benvenga S, degli Uberti EC, Lombardi G, Mantero F, Martino E, Giordano G, Ghigo E: Traumatic brain injury and subarachnoid haemorrhage are conditions at high risk for hypopituitarism: screening study at 3 months after the brain injury. Clin Endocrinol 2004;61:320-326.

33 Dimopoulou I, Kouyialis AT, Tzanella M, Armaganidis A, Thalassinos N, Sakas DE, Tsagarakis S: High incidence of neuroendocrine dysfunction in long-term survivors of aneurysmal subarachnoid hemorrhage. Stroke 2004;35:2884-2889.
34 Hadjivassiliou M, Tooth CL, Romanowski CAJ, Byrne J, Battersby RDE, Osbury S, Crewswell CS, Burkitt E, Stokes NA, Paul C, Mayes AR, Sagar HJ: Aneurysmal SAH: cognitive outcome and structural damage after clipping or coiling. Neurology 2001;56:16721677.

35 Buchanan TW, Kern S, Allen JS, Tranel D, Kirschbaum C: Circadian regulation of cortisol after hippocampal damage in humans. Biol Psychiatry 2004;56:651-656.

36 Robba C, Bacigaluppi S, Bragazzi N, Lavinio A, Gurnell M, Bilotta F, Menon DK: Clinical prevalence and outcome impact of pituitary dysfunction after aneurysmal subarachnoid hemorrhage: a systematic review with metaanalysis. Pituitary 2016;19:522-535.

37 Su H-C, Dai J, Huang X, Zhou W-I, Huang B-X, Cao WL, Sun FK: Classification, diagnosis and treatment of $\mathrm{ACTH}$-independent macronodular adrenal hyperplasia. Can Urol Assoc J 2013;7:594-597.

38 Bosse S, Masciotra V, Solomon C, Stalder T, D'Antono B: Childhood trauma, perceived stress, and hair cortisol in adults with and without coronary artery disease. Psychoneuroendocrinology 2015;61:36.

39 Manenschijn L, Schaap L, van Schoor NM, van der Pas S, Peeters GM, Lips P, Koper JW, van Rossum EF: High long-term cortisol levels, measured in scalp hair, are associated with a history of cardiovascular disease. J Clin Endocrinol Metab 2013;98:2078-2083.

40 Manenschijn L, van Kruysbergen RG, de Jong FH, Koper JW, van Rossum EF: Shift work at young age is associated with elevated long-term cortisol levels and body mass index. J Clin Endocrinol Metab 2011;96:E1862-E1865.

41 Stalder T, Kirschbaum C, Alexander N, Bornstein SR, Gao W, Miller R, Stark S, Bosch JA, Fischer JE: Cortisol in hair and the metabolic syndrome. J Clin Endocrinol Metab 2013;98: 2573-2580.

42 Karlen J, Ludvigsson J, Frostell A, Theodorsson E, Faresjo T: Cortisol in hair measured in young adults - a biomarker of major life stressors? BMC Clin Pathol 2011;11:12.

43 Steiger A, Dresler M, Kluge M, Schüssler P: Pathology of sleep, hormones and depression. Pharmacopsychiatry 2013;46:30-35.

44 Chrousos G, Vgontzas A, Kritikou I: HPA axis and sleep; in De Groot LJ, Beck-Peccoz P, Chrousos G et al (eds): Endotext. South Dartmouth, MDText.com Inc, 2000, 2016.

45 Haugen BR: Durgs that suppress TSH or cause central hypothyroidism. Clin Endocrinol Metab 2009;23:793-800.

46 Fruehwald-Schultes B, Kern W, Oltmanns KM, Sopke S, Toschek B, Born J, Fehm HL, Peters A: Metformin does not adversely affect hormonal and symptomatic responses to recurrent hypoglycemia. J Clin Endocrinol Metab 2001;86:4187-4192.

47 Laakmann G, Hennig J, Baghai T, Schule C: Influence of mirtazapine on salivary cortisol in depressed patients. Neuropsychobiology 2003;47:31-36. 
48 Lenze EJ, Mantella, RC, Shi P, Goate AM, Nowatny P, Butters MA, Andrescu C, Thompson PA, Rollman BL: Elevated cortisol in older adults with generalized anxiety disorder is reduced by treatment: a placebo-controlled evaluation of escitalopram. Am J Geriatr Psychiatry 2011;19:482-490.

49 Granger DA, Hibel LC, Fortunato CK, Kapelewsk CH: Medication effects on salivary cortisol: tactics and strategy to minimize impact in behavioral and developmental science. Psychoneuroendocrinology 2009;34:14371448.
50 Krysiak R, Okopien B: The effect of aggressive rosuvastatin treatment on steroid hormone production in men with coronary artery disease. Bas Clin Pharmacol Toxicol 2014;114: 330-335.

51 Wani TA, Samad A, Tandon M, Saini GS, Sharma PL, Pillai KK: The effects of rosuvastatin on the serum cortisol, serum lipid, and serum mevalonic acid levels in the healthy Indian male population. AAPS Pharm Sci Tech 2010;11:425-432.
52 Edwards KM, Mills PJ: Effects of estrogen versus estrogen and progesterone on cortisol and interleukin-6. Maturitas 2008;61:330333.

53 WHO: International Statistical Classification of Diseases and Related Health Problems. Geneva, WHO, 2005.

54 Stetler C, Miller GE: Depression and hypothalamic-pituitary-adrenal activation: a quantitative summary of four decades of research. Psychosom Med 2011;73:114-126. 